\title{
Cholecystectomy after endoscopic retrograde cholangiopancreatography - effect of time on treatment outcomes
}

\author{
Justyna Kostro ${ }^{1}$, Iwona Marek ${ }^{2}$, Rafał Pęksa ${ }^{3}$, Dariusz Łaski ${ }^{1}$, Andrzej R. Hellmann ${ }^{1}$, Jarek Kobiela ${ }^{1}$, \\ Stanisław Hać ${ }^{1}$, Joanna Pieńkowska ${ }^{4}$, Krystian Adrych ${ }^{5}$, Zbigniew Śledziński ${ }^{1}$ \\ ${ }^{1}$ Department of General, Endocrine and Transplant Surgery, Medical University of Gdansk, Gdansk, Poland \\ 2Department of Gastroenterology and Hepatology, University Clinical Centre Gdansk, Poland \\ ${ }^{3}$ Department of Pathomorphology, Medical University of Gdansk, Gdansk, Poland \\ ${ }^{4}$ II Department of Radiology, Faculty of Health Sciences, Medical University of Gdansk, Gdansk, Poland \\ ${ }^{5}$ Department of Gastroenterology and Hepatology, Medical University of Gdansk, Gdansk, Poland
}

Gastroenterology Rev 2018; 13 (3): 251-257

DOI: https://doi.org/10.5114/pg.2018.78292

Address for correspondence: Justyna Kostro MD, PhD, Department of General, Endocrine and Transplant Surgery, Medical University of Gdansk, 17 Smoluchowskiego St, 80-214 Gdansk, Poland, phone: +48 503192 530, e-mail: kostro@gumed.edu.pl

Cholelithiasis is one of the most common diseases worldwide. It is also a cause of at least one million hospitalisations and more than 700,000 surgical procedures annually in the USA [1]. Although the mortality rate for this disease is relatively low $(0.6 \%)$, it is associated with the possibility of a variety of complications [1]. Choledocholithiasis occurs in $10-18 \%$ of patients with cholelithiasis and varies according to age [2]. Nearly $55 \%$ of patients are symptomatic, and half of those experience complications [3]. Currently, laparoscopic cholecystectomy (LC) is the gold standard treatment for cholecystolithiasis, while endoscopic retrograde cholangiopancreatography (ERCP) with endoscopic sphincterotomy (ES) is the approach commonly used to clear stones from the common bile duct (CBD). There are still discussions about the order in which ERCP and LC should be performed, as well as the timing between the two procedures. Most authors share the opinion that LC should be performed shortly after ERCP [4-9]. A randomised trial has suggested that early LC (within $72 \mathrm{~h}$ ) appears to be safe and may prevent the majority of biliary events in the period following sphincterotomy [10]. A prospective, randomised, multicentre trial confirmed that a wait-and-see policy after endoscopic sphincterotomy combined with cholecysto-choledocholithiasis cannot be recommended as a standard approach after cholecystectomy [11]. Moreover, a delay of LC has been correlated with a higher incidence of cholangitis. Patients with infected bile ducts can develop complications, such as cholecystitis or recurrent choledocholith- iasis [12]. An analysis of histopathological data, combined with surgical outcomes, can be performed before any definitive conclusions can be made [13].

The aim of the present study was to estimate the impact of timing between the procedures and the indication for ERCP in terms of cholecystectomy, postoperative complications, and gallbladder inflammation.

The medical records of patients treated in our hospital for symptomatic CBD stones per 6 years were retrospectively analysed. All patients who underwent ERCP and cholecystectomy in our hospital were analysed. The patients were divide into two groups: the patients in group A had complications such as pancreatitis, cholangitis, Mirizzi's syndrome, acute cholecystitis, and repeated ERCP before $L C$, and the patients in group $B$ had uncomplicated cholecysto-choledocholithiasis. Both groups were divided into two subgroups based on the time elapsed from ERCP to LC. Subgroups 1 (A1 and B1) underwent LC between $72 \mathrm{~h}$ and 6 weeks after ERCP, while subgroups 2 (A2 and B2) underwent LC more than 6 weeks post-ERCP. Patients who underwent surgery less than $72 \mathrm{~h}$ from onset (3 emergency operations) and with pancreatobiliary malignancy (1 case) were excluded from this study.

Data, including age, gender, operation details (time and type of operation, conversion rate, intraoperative complications), median postoperative hospital stay, blood studies (white blood cell count, total serum bilirubin, ALP, ALT, AST, GGT, CRP levels), ultrasound and ERCP findings, and time interval between ERCP and LC, were analysed. 
A histopathological assessment was also performed to determine the severity of the gallbladder wall inflammation. The degree of change was assessed based on the scoring system described by Barcia [9].

Statistical analysis was performed with Statistica Software (StatSoft Polska, Kraków, Polska). T-tests and Mann-Whitney $U$ tests were used to compare the study groups. Statistical significance was determined at $p<0.05$.

During 6 years, 70 patients (female -38 , male -32 ) with a median age of 59.6 years (ranging from 24 to 89 years of age) underwent ERCP for symptomatic CBD stones, followed by cholecystectomy. Group A (1/2) consisted of 28 patients (14/14), and group B (1/2) consisted of $42(19 / 23)$ patients. The studied groups did not differ in terms of age and sex $(p<0.05)$.

All patients underwent ultrasonography (US) and laboratory tests before ERCP. Using US, the presence of stones (single or multiple), choledocholithiasis, cholestasis features, widening of the biliary tree, and cholecystitis were assessed. There were no observed differences between groups $\mathrm{A} 1$ and $\mathrm{A} 2$ or between groups $\mathrm{B} 1$ and B2. However, a statistically significant difference was observed when comparing groups A and B. Cholestasis, cholecystitis, and multiple gallstones were found more often in group A than in group $B$. The patient characteristics are shown in Table I. There was no difference in the lab work between groups A and B in terms of the time between procedures. The results are presented in Table II. During ERCP, all 70 patients underwent sphincterotomy and stone extraction. There was no statistical difference in the number of prostheses placed between both groups (A/B - 23/30). All 28 patients in group $A$ had complications before undergoing ERCP. These complications were recognised either before or during ERCP: 11 patients had acute pancreatitis, nine patients had cholangitis, five patients had Mirizzi's syndrome (type I according to the Csendes classification) [13], 12 patients had acute cholecystitis, and seven patients had more than one ERCP performed before undergoing LC. Patients with acute pancreatitis underwent LC at a significantly later time, more than 6 weeks after ERCP $(p<0.05)$. The other causes did not influence the timing of LC in this group (ns). The 42 patients from group B underwent ERCP because of symptomatic CBDS.

In group $A$, only two operations were performed in an acute setting: one from $A 1$ and one from $A 2$. Group B1 had more operations performed in an acute setting compared to group B2 (7/1). Forty-six laparoscopic

Table I. Ultrasonography and ERCP features

\begin{tabular}{|c|c|c|c|c|}
\hline Features & $\begin{array}{c}\text { A1/A2 } \\
P \text {-value }\end{array}$ & $\begin{array}{c}\text { B1/B2 } \\
P \text {-value }\end{array}$ & $\begin{array}{c}\text { A1/B1 } \\
P \text {-value }\end{array}$ & $\begin{array}{l}\text { A2/B2 } \\
P \text {-value }\end{array}$ \\
\hline Stones in gallbladder & NS & NS & NS & NS \\
\hline Stones single vs. multiple & NS & NS & $<0.05$ & $<0.05$ \\
\hline Stones in biliary tract & NS & NS & $<0.05$ & $<0.05$ \\
\hline Cholestasis & NS & NS & $<0.05$ & $<0.05$ \\
\hline Common bile duct $>6 \mathrm{~mm}$ & NS & NS & NS & NS \\
\hline Cholecystitis & NS & NS & NS & $<0.05$ \\
\hline Biliary prothesis & NS & NS & NS & NS \\
\hline
\end{tabular}

Table II. Results of laboratory tests performed before ECPW

\begin{tabular}{|c|c|c|c|c|c|c|c|c|}
\hline Parameter & $\mathrm{A} 1$ & $\mathrm{~A} 2$ & $P$-value & B1 & B2 & $P$-value & $\begin{array}{c}\text { A1/B1 } \\
P \text {-value }\end{array}$ & $\begin{array}{c}\text { A2/B2 } \\
P \text {-value }\end{array}$ \\
\hline Bilirubin [mg/dl] & 4.3 & 6.1 & NS & 3.7 & 3.9 & NS & NS & NS \\
\hline $\mathrm{AST}[\mathrm{U} / \mathrm{l}]$ & 281.3 & 149.8 & NS & 97.8 & 147.5 & NS & NS & NS \\
\hline $\mathrm{ALT}[\mathrm{U} / \mathrm{I}]$ & 275.4 & 284.9 & NS & 177.4 & 261.6 & NS & NS & NS \\
\hline GGTP $[U / I]$ & 536.5 & 515.0 & NS & 546.1 & 478.2 & NS & NS & NS \\
\hline FALK $[U / I]$ & 289.6 & 204.9 & NS & 245.4 & 198.2 & NS & NS & NS \\
\hline $\mathrm{CRP}[\mathrm{mg} / \mathrm{ll}]$ & 41.2 & 20.6 & NS & 77.7 & 30.3 & NS & NS & NS \\
\hline WBC [G/I] & 8.9 & 7.4 & NS & 7.9 & 7.2 & NS & NS & NS \\
\hline
\end{tabular}


$(A-11 / 9, B-10 / 16)$ and 24 open $(A-3 / 5, B-9 / 7)$ cholecystectomies were performed. Conversion to open surgery was necessary in 16 cases (A - 1/5, B - 4/5). There was a significantly $(p<0.05)$ higher conversion rate in group $A 2$ compared to group $A 1$. The reasons for conversion in group A were inflammatory infiltration and adhesions surrounding the gall bladder. There was no difference observed in the frequency of conversions between groups B1 and B2. The reason for the three conversions was an empyema of the gallbladder, while the remaining conversions were performed due to infiltrative inflammation or adhesions. In all cases, the reason for conversion was impossibility of identifying the structures of Calot's triangle. The median operative time in group $A$ was $85 \mathrm{~min}$, while in group $B$ it was 60 minutes (range $A 1: 50-90, A 2: 40-150$, and $B 1$ : 30-150, B2: 30-190 min). Operations in group $B$ (B1 and B2) were simpler, and extending the time during open surgery did not affect the average operation time. Most of the operations performed after 6 weeks had longer duration. The details of intraoperative complications are summarised in Table III.

The postoperative complications in group A1 were two wound infections and one bleeding and bile leak with reoperation, while in group $A 2$ there were no observed complications. In group B1, one wound infection with eventration was reported, while in group B2, there was one wound infection, one case of pneumonia, one case of bile leakage with ERCP, and one inflammatory infiltration to the liver. The media postoperative hospital stay was 2 days in all groups (range: A1: 1-20, A2: 1-5, B1: 1-14, B2: 1-12 days). All operations were performed by specialists using the standard four-port technique. All patients received a single dose of prophylactic antibiotics before the procedure.
Histopathological examination confirmed the presence of inflammation in all cases. Acute inflammation of the gallbladder was observed in 26 cases (A1/A2 - 8/4, $B 1 / B 2-10 / 4)$, and chronic inflammation was observed in 44 cases (A1/A2 - 6/10, B1/B2 - 9/19) (Figure 1).

More cases of acute inflammation of the gallbladder were observed in group A1 (57\%) and B1 (53\%), whereas chronic inflammation was observed more often after 6 weeks post-ERCP in both groups (A2 - 71\%, B2 - 83\%). Inflammatory activity was present in $42 \%$ of the cases in group A1 (25\% with mild, $8 \%$ with moderate, and $8 \%$ with severe activity), in $33 \%$ of the cases in group A2 $25 \%$ with mild and $8 \%$ with moderate activity), in $71 \%$ of the cases in group B1 ( $41 \%$ with mild and $29 \%$ with severe activity), and in $24 \%$ of the cases in group B2 (14\% with mild and 10\% with severe activity) (Figure 2).

Fibrosis was observed in all cases (A1 - 33\% mild, $33 \%$ moderate, $33 \%$ severe; $A 2-50 \%$ mild, 33\% moderate, $17 \%$ severe; B1 - 18\% mild, $29 \%$ moderate, $53 \%$ severe; B2 - 48\% mild, 38\% moderate, $14 \%$ severe). A moderate or severe degree of fibrosis showed a positive correlation with conversion for open surgery (Figure 3).

The degree of inflammatory mononuclear infiltrate in group A1 was moderate in $17 \%$ of cases and severe in $83 \%$, while in group $\mathrm{A} 2$, the occurrence was moderate in $8 \%$ of cases and severe in $92 \%$. In group B1, inflammatory mononuclear infiltrate was present in $94 \%$ of cases (mild in $6 \%$, moderate in $18 \%$, and severe in $70 \%$ ), while group B2 had an occurrence of $90 \%$ (mild in $5 \%$, moderate in $19 \%$, and severe in 67\%) (Figure 4).

Only group B showed statistically significant differences in the histopathological assessment of changes taking place in the course of cholecystitis $(p<0.05)(\mathrm{Ta}-$ ble IV).

Table III. Surgery - operation time, \% of elective, laparoscopic and converted procedures, intraoperative findings, and postoperative complications rate

\begin{tabular}{|c|c|c|c|c|}
\hline Parameter & $\mathrm{A} 1 / \mathrm{A} 2$ & $P$-value & B1/B2 & $P$-value \\
\hline Median operation time [min] & $90 / 80$ & NS & $60 / 60$ & NS \\
\hline Elective surgery \% & $93 / 93$ & NS & $63 / 96$ & $<0.05$ \\
\hline Operation type - laparoscopic, $n$ (\%) & $79 / 64$ & NS & $53 / 70$ & NS \\
\hline Conversion rate, $n(\%)$ & $6 / 36$ & $<0.05$ & $21 / 22$ & NS \\
\hline Median postoperative hospital stay [days] & $2 / 3$ & NS & $2 / 2$ & NS \\
\hline \multicolumn{5}{|l|}{ Intraoperative findings, $n(\%)$ : } \\
\hline Cholecystitis & $71 / 57$ & NS & $74 / 30$ & NS \\
\hline Empyema & $0 / 0$ & NS & $32 / 13$ & $<0.05$ \\
\hline Adhesions & $41 / 71$ & NS & $74 / 61$ & NS \\
\hline Postoperative complication rate, $n(\%)$ & $12 / 0$ & $<0.05$ & $5 / 17$ & $<0.05$ \\
\hline
\end{tabular}




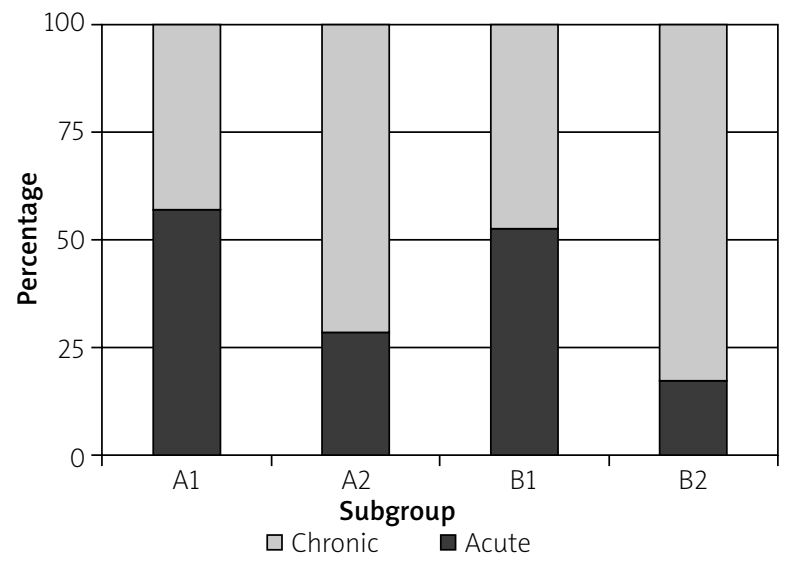

Figure 1. Proportion of acute and chronic inflammation type in all subgroups

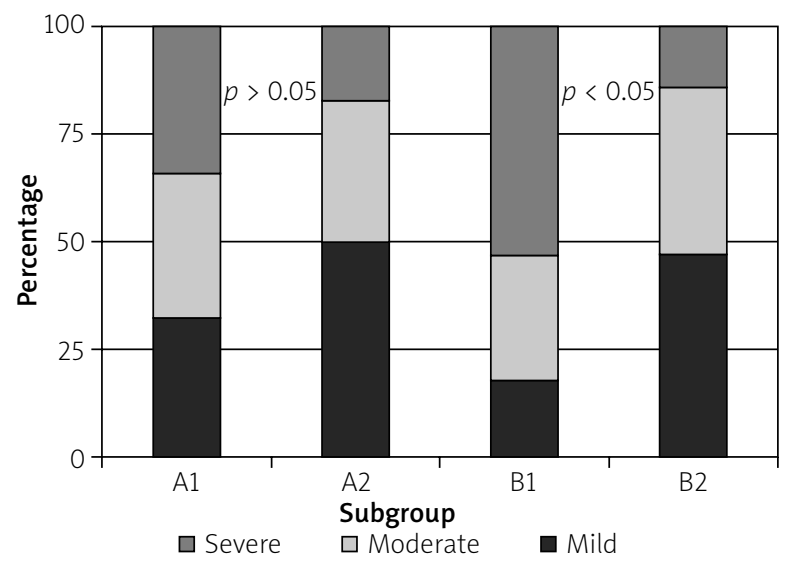

Figure 3. Proportion of different fibrosis stages in all subgroups

The presence of stones in the common bile duct and the gallbladder is an indication to perform ERCP and LC. There is currently no unanimous opinion regarding the timing of these procedures. This publication discusses the results of cholecystectomy in patients who underwent ERCP earlier because of cholecysto-choledocholithiasis. Patients were divided into two groups, based on their indications for ERCP and the time elapsed between ERCP and LC. Most of the work presented excludes pa-

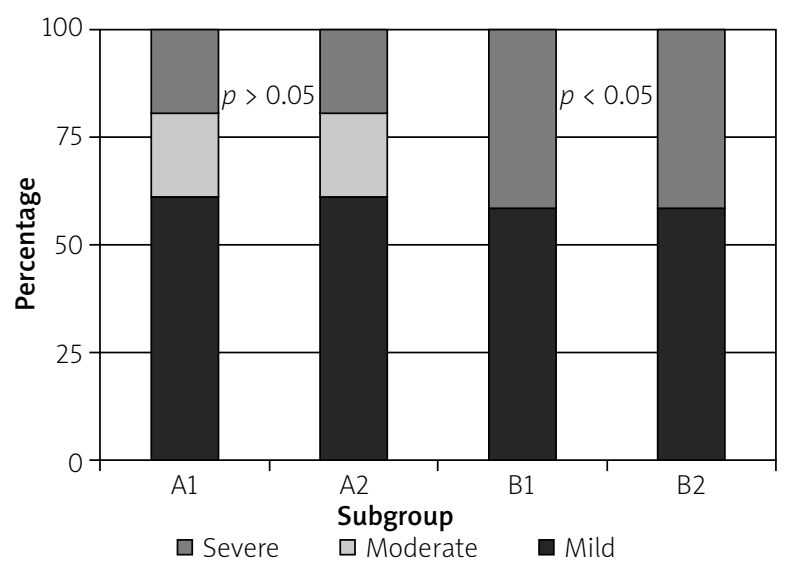

Figure 2. Proportion of different inflammatory activity stages in all subgroups

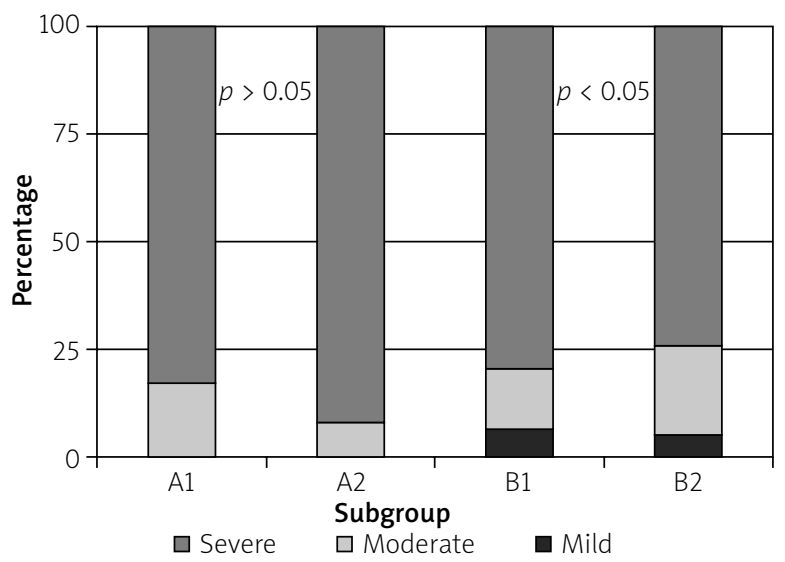

Figure 4. Proportion of different degrees of inflammatory mononuclear infiltrate in all subgroups

tients with complications occurring before ERCP, presenting the effect of time on the final outcome of LC [2-4, 10]. In our material, patients from group A showed coexisting illnesses, such as pancreatitis, cholangitis, Mirizzi's syndrome, acute cholecystitis, or repeated ERCP, before LC was performed, as in the data from Waisberg et al. [14]. The American Society for Gastrointestinal Endoscopy (ASGE) guidelines confirm a high or very high probability of the presence of stones in the CBD [15]. Accord-

Table IV. Histopathological examination findings

\begin{tabular}{|c|c|c|c|c|}
\hline Parameter & $\begin{array}{c}\text { A1/A2 } \\
P \text {-value }\end{array}$ & $\begin{array}{c}\text { B1/B2 } \\
P \text {-value }\end{array}$ & $\begin{array}{l}\text { A1/B1 } \\
P \text {-value }\end{array}$ & $\begin{array}{l}\text { A2/B2 } \\
P \text {-value }\end{array}$ \\
\hline Chronic inflammation & NS & NS & NS & NS \\
\hline Inflammatory activity & NS & $<0.05$ & NS & NS \\
\hline Fibrosis & NS & $<0.05$ & NS & NS \\
\hline Acute vs. chronic inflammation & NS & $<0.05$ & NS & NS \\
\hline
\end{tabular}


ing to the ASGE, all patients demonstrated strong or very strong predictors of the risk of CBDS and, therefore, first underwent ERCP. Guidelines on the management of CBDS were also commissioned by British gastroenterologists, surgeons, and radiologists. They recommend that endoscopists ensure adequate biliary drainage is achieved in patients with CBDS that have not been extracted. The short-term use of a biliary stent, followed by further endoscopy or surgery, is advocated [16]. Deferred operation in the case of minor deposits in the gallbladder was an indication for stent implantation into the bile duct. In our material, a high percentage of patients required the prosthesis to be left in the $\mathrm{CBD}$, but this procedure had no observable effects on the histopathological examinations or the evaluation of the $L C$ result. Following ERCP (combined with ES or biliary stenting), cholecystectomy was performed. Some cases required emergency operations despite elective LC being the preferred method, and in some cases it was necessary to consider both LC and ERCP as the same time. The interval between LC and ERCP may vary from hours to months. Simultaneous LC and laparoscopic common bile duct exploration (LCBDE) can result in a shorter hospitalisation duration and, thus, greater patient acceptance [17]. The British group concluded that a single surgical procedure for common bile duct stone disease is feasible, cost-effective, and should be available for most patients [17]. This method, however, has intrinsic limitations because it is not possible to perform LCBDE in most hospitals. The alternative is surgical laparoendoscopic rendezvous (LERV) with LC and ERCP. Studies have proven that this modified technique can effectively reduce the surgical time, as well as the endoscopic part of LC+ ERCP/LERV, compared with the traditional technique [18]. In the management of cholelithiasis and concomitant choledocholithiasis, LERV reduces post-ERCP pancreatitis, reduces hospitalisation stay, and increases patient compliance $[19,20]$. However, from a logistical standpoint, it can be difficult to synchronise those two procedures [2]. Despite its many advantages, this procedure cannot be considered as the "gold standard". Not every case is classified as urgent, and in many cases it is advisable to perform ERCP first and then LC (e.g. due to high levels of bilirubin or other complications associated with CBDS). In patients who present with complications, such as cholangitis or pancreatitis, simultaneous LC and ERCP should not be indicated. Data has sown that patients with endoscopic clearance of choledocholithiasis, particularly after endoscopic sphincterotomy, should receive elective laparoscopic cholecystectomy within 6 weeks after a cholangitic attack [4]. The late surgery group had significantly more intraoperative and postoperative complications compared with the early surgery group [4]. In our study, group A2 patients with cholangitis were statistically more likely to be converted to the open approach and to have adhesions reported. Postoperative complications were observed only in group $A 1$, which resulted in prolonged hospitalisation. In this case, however, the patient had cholangitis, cholecystitis and Mirizzi's syndrome, and a bilirubin level of $16.6 \mathrm{mg} \%$ before ERCP. The patient initially underwent the classical surgery. Bile leakage and postoperative bleeding were associated with increased inflammatory reaction around Calot's triangle. The histopathological assessment revealed greater inflammatory activity in groups A1 and B1. The timing of cholecystectomy in gallstone pancreatitis remains controversial and is dependent upon the severity of pancreatitis and the patient's condition. An early LC may be safely performed for patients with mild gallstone pancreatitis. The practice of delaying $\mathrm{LC}$ until receiving normal laboratory values in mild disease cases appears to be unnecessary [21]. However, the decision concerning the optimal time to perform surgery should be considered individually, depending on the severity of inflammation. We can still expect to observe worse outcomes in patients with severe pancreatitis. In the presented material, eight patients underwent surgery more than 6 weeks after ERCP, and only three patients with mild pancreatitis underwent surgery within 6 weeks of ERCP. Delayed surgery caused persistent severe pancreatitis. In group A2, LC with conversion was performed more often than in group A1 (2/0), and both operations were performed 16 weeks after ERCP. However, the remaining data from the groups is comparable. It seems reasonable to perform LC as soon as possible following the improvement of a patient with severe acute pancreatitis. In our opinion delaying surgery may result in difficulties and postoperative recurrent acute pancreatitis, especially when small multiple stones in the bladder were diagnosed. In the case of Mirizzi's syndrome, consideration is important not only for the time of the ERCP but also the feasibility of safe laparoscopic surgery. Large inflammatory infiltration within Calot's triangle and the possibility of the coexistence of a fistula may force a surgeon to perform open cholecystectomy conversion. It seems, however, that in type I (according to the Csendes classification), laparoscopic surgery is safe [14]. In our material, apart from a previously commented case, there was only one necessary conversion to open surgery (in a patient in group A2), in a case with coexisting acute pancreatitis. Another problem is the timing of LC before ERCP in patients with acute cholecystitis. Some believe that early LC $(<72 \mathrm{~h})$ is safe and feasible for acute cholecystitis, with the additional benefit of shorter total hospital stays [22] and that LC performed within 2 days of the presentation of acute cholecystitis yielded the best outcomes associ- 
ated with fewer complications, a lower mortality rate, and the lowest costs [23, 24]. However, $44 \%$ of patients had acute symptoms of cholecystitis for 3 or more days before being admitted to the hospital, making it difficult to operate shortly after the beginning of the disease. The 72-hour period for early LC can be extended to 7 days, according to the research of Soffer et al., in which the authors did not find any statistical differences in the complication or conversion rates within the first 7 days of gallbladder inflammation [25]. However, the postponement of surgery may result in difficulty in identifying Calot's triangle due to inflammatory infiltration [23]. Surgeons who perform cholecystectomies should be familiar with different techniques, such as "fundus-first" dissection, "hydrodissection" with a suction-irrigation device, early decompression of empyema or hydrops of the gallbladder, and prettied loop ligation of cystic duct stump $[22,26]$. In our material, delaying LC after ERCP with cholecystitis was related to a higher conversion rate than in early LC (3 to 1). A single-stage procedure is recommended to manage symptomatic gallstones and choledocholithiasis when local resources and expertise permit [27]. Both methods seem to be safe and acceptable in this situation. LC associated with intraoperative ERCP, according to the rendezvous technique, can reduce the incidence of complications, decrease the length of stay, and reduce surgery times and costs [28]. If sequential treatment is chosen, the question of the length of time between endoscopic intervention and LC is still debatable. It is proposed that surgeons perform LC early, up to $72 \mathrm{~h}$ from ERCP [10, 22]. Many articles have compared early LC (up to $72 \mathrm{~h}$ ) with delayed LC (up to 6-10 weeks), and they have proven that early LC has a lower conversion rate, fewer postoperative complications, and shorter hospital stays, with comparable operating times and overall complications [3, 6, 11, 23, 29]. Early LC may also prevent recurrent biliary complications, which are associated with increased postoperative morbidity and prolonged hospital stays $[4,6,10,12]$.

In this study, the median operation time and postoperative hospital stay were similar, regardless of the time between procedures. Statistically, more patients underwent surgery in the acute setting in group B1. Furthermore, the intraoperative complication rate was higher in group B1. Acute gallbladder inflammation and empyema were observed and hindered the execution of the procedure. Most of these operations were performed classically or required conversion. De Vries et al. have reported the tendency of a higher conversion rate of a laparoscopic procedure after 2-6 weeks post-ERCP [29]. In group B1, the activity and severe fibrosis were higher, which could cause intraoperative difficulties. This finding may imply that operations performed with- in 3 days $(<72 \mathrm{~h}$ ) of ERCP could protect patients from emergency surgery and allow for laparoscopic surgery. The histological evaluation demonstrated a higher rate of chronic cholecystitis, as well as lower activity and primarily mild fibrosis. None of the patients with mild fibrosis required conversion to the open method. The effect of chronic inflammation was not noticed in terms of the difficulty of the procedure and the related number of conversions.

Given the literature and the analysis of the data in this study, we believe that it is worth accelerating LC following ERCP. In terms of the accompanying complications, LC should be performed after stabilising the patient. Early laparoscopic cholecystectomy after ERCP for common bile duct disease could be an answer to reducing conversion rates and complications.

\section{Conflict of interest}

The authors declare no conflict of interest.

\section{References}

1. Stinton LM, Shaffer EA. Epidemiology of gallbladder disease: cholelithiasis and cancer. Gut Liver 2012; 6: 172-87.

2. Ding YB, Deng B, Liu XN, et al. Synchronous vs sequential laparoscopic cholecystectomy for cholecystocholedocholithiasis. World J Gastroenterol 2013; 19: 2080-6.

3. Salman B, Yilmaz U, Kerem M, et al. The timing of laparoscopic cholecystectomy after endoscopic retrograde cholangiopancreaticography in cholelithiasis coexisting with choledocholithiasis. J Hepatobiliary Pancreat Surg 2009; 16: 832-6.

4. Li VK, Yum JL, Yeung YP, et al. Optimal timing of elective laparoscopic cholecystectomy after acute cholangitis and subsequent clearance of choledocholithiasis. Am J Surg 2010; 200: 483-8.

5. Reinders JSK, Gouma DJ, Heisterkamp J, et al. Laparoscopic cholecystectomy is more difficult after a previous endoscopic retrograde cholangiography. HPB 2013; 15: 230-4.

6. Schiphorst AH, Besselink MG, Boerma D, et al. Timing of cholecystectomy after endoscopic sphincterotomy for common bile duct stones. Surg Endosc 2008; 22: 2046-50.

7. Rubago LR, Vicente C, Soler F, et al. Two-stage treatment with preoperative endoscopic retrograde cholangiopancreatography (ERCP) compared with single-stage treatment with intraoperative ERCP for patients with symptomatic cholelithiasis with possible choledocholithiasis. Endoscopy 2006; 38: 779-86.

8. Morino M, Baracchi F, Miglietta C, et al. Preoperative endoscopic sphincterotomy versus laparoendoscopic rendezvous in patients with gallbladder and bile duct stones. Ann Surg 2006; 244: 889-96.

9. Barcia JJ. Histologic analysis of chronic inflammatory patterns in the gallbladder: diagnostic criteria for reporting cholecystitis. Ann Diagn Pathol 2003; 7: 147-53.

10. Reinders JS, Goud A, Timmer R, et al. Early laparoscopic cholecystectomy improves outcomes after endoscopic sphincter- 
otomy for choledochocystolithiasis. Gastroenterology 2010; 138: 2315-20.

11. Boerma D, Rauws EAJ, Keulemans YCA, et al. Wait-and-see policy or laparoscopic cholecystectomy after endoscopic sphincterotomy for bile-duct stones: a randomized trial. Lancet 2002; 360: 761-5.

12. Reinders JS, Kortram K, Vlaminckx B, et al. Incidence of Bactobilia increases over time after endoscopic sphincterotomy. Dig Surg 2011; 28: 288-2.

13. Bartosiak K, Liszka M, Drazba T, et al. Unexpected pathological findings after laparoscopic cholecystectomy - analysis of 1131 cases. Videosurgery Miniinv 2018; 13: 62-6.

14. Waisberg J, Corona A, de Abreu IW, et al. Benign obstruction of the common hepatic duct (Mirizzi syndrome): diagnosis and operative management. Arq Gastroenterol 2005; 42: 13-8.

15. Rubin MI, Thosani NC, Tanikella R, et al. Endoscopic retrograde cholangiopancreatography for suspected choledocholithiasis: testing the current guidelines. Dig Liver Dis 2013; 45: 744-9.

16. Williams EJ, Green J, Beckingham I, et al. Guidelines on the management of common bile duct stones (CBDS). Gut 2008; 57: 1004-21.

17. Rogers SJ, Cello JP, Horn JK, et al. Prospective randomized trial of LC_LCBDE vs ERCP/S_LC for common bile duct stone disease. Arch Surg 2010; 145: 28-33.

18. Wei L, Qunwei W, Jing X, et al. A modified technique reduced operative time of laparoendoscopic rendezvous endoscopic retrograde cholangiopancreatography combined with laparoscopic cholecystectomy for concomitant gallstone and common bile ductal stone. Gastroenterol Res Pract 2014; 2014 86129529.

19. Sahoo MR, Kumar AT, Patnaik A. Randomised study on single stage laparo-endoscopic rendezvous (intra-operative ERCP) procedure versus two stage approach (pre-operative ERCP followed by laparoscopic cholecystectomy) for the management of cholelithiasis with choledocholithiasis. J Minim Access Surg 2014; 10: 139-43.

20. Liverani A, Muroni M, Santi F, et al. One-step laparoscopic and endoscopic treatment of gallbladder and common bile duct stones: our experience of the last 9 years in a retrospective study. Surg 2013; 79: 1243-7.

21. Falor AE, de Virgilio C, Stabile BE, et al. Early laparoscopic cholecystectomy for mild gallstone pancreatitis time for a paradigm shift. Arch Surg 2012; 147: 1031-5.

22. Lai PB, Kwong KH, Leung KL, et al. Randomized trial of early versus delayed laparoscopic cholecystectomy for acute cholecystitis. Br J Surg 1998; 85: 764-7.

23. Lau H, Lo CY, Patil NG, et al. Early versus delayed-interval laparoscopic cholecystectomy for acute cholecystitis: a metaanalysis. Surg Endosc 2006; 20: 82-7.

24. Zafar SN, Obirieze A, Adesibikan B, et al. Optimal time for early laparoscopic cholecystectomy for acute cholecystitis. JAMA Surg 2015; 150: 129-36.

25. Soffer D, Blackbourne LH, Schulman Cl, et al. Is there an optimal time for laparoscopic cholecystectomy in acute cholecystitis? Surg Endosc 2007; 21: 805-9.

26. Sahbaz NA, Peker KD, Kabuli HA, et al. Single center experience in laparoscopic treatment of gallbladder perforation. Videosurgery Miniinv 2017; 12: 372-7.
27. Zang JF, Zhang C, Gao JY, et al. Endoscopic retrograde cholangiopancreatography and laparoscopic cholecystectomy during the same session: feasibility and safety. World J Gastroenterol 2013; 19: 6093-7.

28. Kenny R, Richardson J, McGlone ER, et al. Laparoscopic common bile duct exploration versus pre or post-operative ERCP for common bile duct stones in patients undergoing cholecystectomy: is there any difference? Int J Surg 2014; 12: 989-93.

29. De Vries A, Donkervoort SC, Van Geloven AA, et al. Conversion rate of laparoscopic cholecystectomy after endoscopic retrograde cholangiography in the treatment of choledocholithiasis. Does the time interval matter? Surg Endosc 2005; 19: 9961001.

Received: 13.05.2018

Accepted: 12.06.2018 